
\title{
is Research Square \\ CBX3 Regulated by miR-139 Promoted the Development of HCC by Regulating Cell Cycle Progression
}

\section{Pan Zhang}

Luoyang Central Hospital Affiliated to Zhengzhou University

\section{Xiaoyan Yang}

Henan University of Science and Technology

\section{Zhongming Zha}

Luoyang Central Hospital Affiliated to Zhengzhou University

\section{Yumeng Zhu}

Luoyang Central Hospital Affiliated to Zhengzhou University

\section{Guoqiang Zhang}

Luoyang Central Hospital Affiliated to Zhengzhou University

\section{Guotao Li ( $\sim$ lyszxyy2048@163.com )}

Department of Infectious Diseases, Luoyang Central Hospital Affiliated to Zhengzhou University, Luoyang, Henan 475100, China. https://orcid.org/0000-0003-1570-6738

\section{Primary research}

Keywords: HCC, CBX3, miR-139, cell cycle progression

Posted Date: August 21st, 2020

DOl: https://doi.org/10.21203/rs.3.rs-59452/v1

License: (c) (1) This work is licensed under a Creative Commons Attribution 4.0 International License. Read Full License 


\section{Abstract}

Background Hepatocellular carcinoma (HCC), comprises of the major primary liver cancer, is one of the most lethal malignancies in the world [1]. Increasing evidence has demonstrated that chromobox protein homolog 3 (CBX3) functioned as an oncogene in different cancers. However, its expression profiles and biological functions in HCC remain exactly unknown.

Methods Data of CBX3 expression in HCC acquired from TCGA and GEO databases were analyzed. The biological functions of $\mathrm{CBX} 3$ in $\mathrm{HCC}$ were examined by in vitro experiments. Bioinformatics analysis, qRTPCR and western blot were performed to explpore the mechanism of CBX3 involved in HCC.

Results $\mathrm{CBX} 3$ mRNA was upregulated in HCC tissues, and overexpression of $\mathrm{CBX} 3$ mRNA was negatively correlated with malignancies and poor prognosis in HCC patients. Knocking down of CBX3 induced slower growth, less migration and fewer invasions of the HCC cells in vitro. Moreover, bioinformatics analysis and experimental observation indicated that $\mathrm{CBX} 3$ expression was correlated with cell cycle regulation proteins of HCC cells. Finally, Starbase predicted that the miR-139 could directly target CBX3 in HCC; Confirmatory experiments verified that miR-139 overexpression attenuated the HCC cells proliferation and migration, which could be reversed by overexpressing CBX3 concurrently.

Conclusion Our results concluded that miR-139/CBX3 axis may involve in the HCC development through regulating cell cycle progression and may be a promising target in the treatment of HCC.

\section{Introduction}

Hepatocellular carcinoma (HCC) is the sixth malignancy and has ranked as the fourth leading cause of cancer-mortality worldwide [2]. Surgery, such as Liver resection, liver transplantation and liver ablation, may benefit for early liver cancer [3]. However, the five-year survival of HCC is usually less than $30 \%$ given that most people were diagnosed with advanced liver cancer[4]. Therefore, there is an essential need to further study the molecular mechanisms and develop the effective therapeutic targets in HCC.

Chromobox homolog protein 3 (CBX3), corresponds to HP1y, is a member of the heterochromatin protein 1 (HP1) family [5]. CBX3 plays a critical role in many biological processes, such as heterochromatin formation and gene silencing, DNA damage repairing, RNA splicing, transcription activation, stem cell differentiation, and reprogramming of somatic cells[6]. Given its wide distribution and different roles in gene regulation, CBX3 has been reported to be closely involved in development of pancreatic cancer, prostate cancer and breast cancer. However, the mechanisms and roles of CBX3 in HCC are still unclear, which need further study and clarification.

miRNAs are a group of endogenous non-coding small RNAs and could regulate the expression of their target $m R N A s$ by binding to their 3'-untranslated region (3'-UTR)[7]. Mounting evidence has validated that miRNAs play an important role in the pathogenesis, proliferation and other biological processes of 
cancers[8]. Hence, we need to explore whether there is a miRNA that can serve as a post-transcriptional regulator of $\mathrm{CBX} 3$ involved in the development of HCC.

Here our study suggested that CBX3 was overexpressed and performed as a poor prognostic marker in HCC. We also identified that depression of CBX3 inhibited the progression of HCC in vitro. Further bioinformatics analysis and validation experiments confirmed that CBX3 promoted the development of $\mathrm{HCC}$ by regulating cell cycle and played as a direct target of miR-139 involving in the progression of HCC. In conclusion, miR-139/CBX3 axis involved in the progression of HCC through regulating cell cycle progression.

\section{Materials And Methods}

\section{Collection of public HCC database}

Differential gene expression profiles in HCC and non-tumor liver tissues were acquired from the Cancer Genome Atlas (TCGA) (http://tcga-data.nci.nih.gov/) [9], and the survival data of HCC patients was also collected and analyzed. LIRIJP (Liver Cancer-RIKEN, JP project) dataset was obtained from the International Cancer Genome Consortium (ICGC) and 18 liver cancer mRNA microarray data sets were acquired from the Gene Expression Omnibus (GEO) databases (http://www.ncbi.nlm.nih.gov/geo). The difference expression between non-tumorous tissues and HCC were identified by the BRB-array tool.

\section{Tissue microarray (TMA)}

The TMA was composed of $341 \mathrm{HCC}$ tissues and corresponding adjacent non-tumor tissues (obtained from the 1st Affiliated Hospital of Zhengzhou University between 2011 and 2015), all contained complete clinic-pathological information. This study has been formal approval of the ethics committee in the $1 \mathrm{st}$ Affiliated Hospital of Zhengzhou University. All patients signed written informed consent.

\section{Immunohistochemistry (IHC) analysis}

Briefly, xylene and graded alcohol were used to deparaffinize and rehydrate the sample sections, respectively. After antigen retrieved and blocking with bovine serum albumin, then the sections were placed at $4^{\circ} \mathrm{C}$ temperature with the primary antibodies CBX3 (dilution 1:100, Proteintech Group, Wuhan, China) overnight. Following, the goat anti-rabbit secondary antibodies were added for incubation at normal temperature, and the slides were counterstained with hematoxylin. The NanoZoomer 2.0-RS system was applied to observe the imaging of $\mathrm{IHC}$ under Hematoxylin and Eeosin staining. Two experienced pathologists scored the $\mathrm{CBX} 3$ staining in an independent and blinded manner. Sections were semi-quantitatively scored for the CBX3 staining patterns as follows: the staining extent in each core was scored as $1+(<25 \%$ staining of tumor cells), $2+(25 \%-50 \%$ staining of tumor cells), $3+(50 \%-75 \%$ staining of tumor cells) or $4+(>75 \%$ staining of tumor cells. Scores of $3+$ and $4+$ were defined as exhibiting high expression and scores of $1+$ and $2+$ were deemed as exhibiting low expression

\section{Cell culture}


HCC cell lines (Hep3B and SMMC-7721) and immortalized human normal liver cell lines (L02 and Changliver) were purchased from the Cell Bank of Institutes for Biological Sciences (Shanghai, China). Cells were cultured at stationary $37^{\circ} \mathrm{C}$ in Dulbecco's modified Eagle's medium (Gibco, NY, USA)) with $10 \%$ fetal bovine serum in an atmosphere of $5 \% \mathrm{CO} 2$. All above cell lines in culture had been passaged for less than 6 months when our experiments began.

\section{Polymerase chain reaction (PCR) assays and immunoblotting analysis}

Trizol reagent (Invitrogen, Carlsbad, CA, USA) was applied to collect total RNA in accordance with the manufacturer's instructions. Quantitative reverse transcription-PCR (RT-qPCR) was performed following manufacturer's protocol. Actin or U6 expression was used to normalize the target genes' relative expression levels. The $2^{-\Delta \Delta C t}$ method was used to analyze the data. Every experiment was performed in three times independently.

RIPA protein extraction reagent (KeyGEN, Nanjing, China) was used to extract proteins from the whole cells on ice, Each groove of $12 \%$ SDS- PAGE gels was loaded in equivalent proteins, after electrophoresis, proteins were transferred to polyvinylidene difluoride (PVDF) membranes (Millipore, USA). After blocking, the membranes were incubated in 1:1000 anti- CBX3, 1:2000 anti-c-Myc, 1:500 anti-E2F, 1:1000 anti-CCK1, 1:1000 anti-cyclin D1, 1:1000 anti- $\beta$-actin for overnight at $4^{\circ} \mathrm{C}$. Following, the membranes were placed with goat anti-mouse secondary antibodies (1:1000; Abcam) at normal temperature for two hours. Bands were visualized and analyzed by the Odyssey infrared imaging system and Odyssey 3.0 software (Thermo Scientific), respectively.

\section{Transfection}

Three CBX3-target siRNAs (si- CBX3-1, si- CBX3-2 and si- CBX3-3) were utilized to knock down CBX3; a non-silencing siRNA oligonucleotide (si-NC) was a negative control (Invitrogen, CA, USA). CBX3 CDNA was subcloned into a pcDNA3.1 vector as a plasmid (obtained from Invitrogen, CA, USA) to overexpress CBX3, an empty vector was used to be a negative control (pcDNA3.1-NC). MiR-139 mimics, miR-139 inhibitor and the respective negative controls (mimics-NC, inhibitor-NC) and miR-NC were synthesized by GenePharma (Shanghai, China). For transient transfection, HCC cell lines were transfected using Lipofectamine 3000 (Invitrogen, Carlsbad, USA) in line with the manufacturer's guidelines.

\section{Dual-Luciferase reporter assay}

Both wild-type and mutant variant 3'-UTR fragment of CBX3 mRNA were cloned into pmirGLO vectors (Promega, Madison, WI, USA) named as Wt-CBX3 3'-UTR and Mut-CBX3 3'-UTR, respectively. Then Hep3B and SMMC-7721 cells were co-transfected with Wt-CBX3 or Mut-CBX3 vectors and miR-139 mimics or miR-NC with Lipofectamine 3000 reagent (Invitrogen). 48h for transfection later, the Dual-Luciferase Reporter Assay System was used to analyze the luciferase activity (Promega, Madison, USA). The results for experiments are showed as the mean value \pm SD in three times. 


\section{Cell growth assay}

Cell Counting Kit-8 (CCK-8) assay was applied to examine cell growth [10]. Transfected and nontransfected cells were cultured in six 96-well plates. Then the 96-well microplates were put into the incubator. $10 \mathrm{ml} \mathrm{CCK}-8$ reagent was added to each well in a 96-well plate every 24 hours for 5 days, after which the plate was incubated for 2 hours at $37^{\circ} \mathrm{C}$. Subsequently, the absorbance was record at $450 \mathrm{~nm}$ by using a spectrophotometer (Molecular Devices, CA, USA).

Colony formation assay was performed to evaluate HCC cell growth. 1,000 cells per well were placed into the 6-well plates. After 14 days for incubation, cells were fixed with paraformaldehyde (4\%) and stained with $2 \%$ crystal violet, then counted and imaged.

\section{Invasion and wound healing assay}

Wound healing assays were utilized to confer the migration ability of HCC cells. Hep3B and SMMC-7721 cells were seeded into the six-well plate, when the growth density reached approximately $90 \%$ confluences, we made a gap using a pipette tip of $10 \mu \mathrm{L}$. And the gaps were taken photos at 0,24 and 48 hours later with the microscope (magnification, $\times 100$ ).

Transwell assay was performed to check the cell invasion ability. $1 \times 10^{4}$ indicated cells were plated into the transwell insert (the upper chamber) with serum-free medium, DMEM with $10 \%$ FBS was filled in the bottom chamber. 24 hours later, the invasive cells on the bottom chamber were fixed and stained. Finally, the migratory cells were imaged and counted.

\section{Bioinformatics analysis}

Starbasewas used to predict that miR-139 was binding to 3'UTR of CBX3mRNA. Gene set enrichment analysis (GSEA) and the Kyoto Encyclopedia of Genes and Genomes (KEGG) analysis were utilized to elucidate the individual genes sets associated with CBX3 expression in the LIHC TCGA data, $\mid$ NES| $>1$, FDR q value $<0.25$ and P-value $<0.05$ were considered as significance set-point for GSEA. Moreover, gene set variation analysis (GSVA) was performed to further identify significant differences in genomeregulated biological processes.

\section{Statistical analysis}

We analyzed the data by using the SPSS software (version 23.0, SPSS Inc., Chicago, IL) and GraphPad Prism 6.0 (GraphPad Software, Inc., LaJolla, CA, USA). The association of CBX3 expression with clinicpathologic parameters in HCC was verified using the $\chi^{2}$ test. The continuous variables were calculated by Mann-Whitney $U$ test and unpaired $t$ test. Pearson's correlation test was applied to analyze the correlation between CBX3 and Ki-67 mRNA expression, CBX3 and PCNA expression, CBX3 and miR139 mRNA expression. The log-rank tests and Kaplan-Meier methods were applied to evaluate the Patient survival. $P$ $<0.05$ was identified as statistical significance level for all analysis 


\section{Results}

\section{CBX3 was usually unregulated and acted as an unfavorable prognostic marker in HCC}

To identify the CBX3 expression profiles in HCC, the TCGA, LIRIJP and GEO databases were extracted and analyzed. Figure 1A showed that the expression level of CBX3 mRNA was commonly upregulated in HCC compared with that in normal liver tissues. Moreover, analysis of GSE6764 (Figure 1B) revealed that CBX3 mRNA expression was overexpressed in HCC tissues compared with non-tumor liver tissues (early HCC or advanced HCC vs average of normal liver, cirrhotic, low grade dysplastic liver and high grade dysplastic liver, all $P<0.001$ ). Analysis of GSE84598 (Figure 1C) exhibited that CBX3 was elevated expressed in HCC tissues than that in normal liver tissues $(P<0.01)$ and liver border tissues $(P<0.05)$. As shown in Figure 1D, the expression level of CBX3 mRNA was markedly higher in $\mathrm{HCC}(P<0.01)$ and portal vein tumor thrombosis $(P<0.01)$ than in normal liver tissues. To further study the clinical prognostic significance of CBX3 mRNA overexpression in HCC, we extracted the information of pathological parameters and patient survival from TCGA. We acquired that CBX3 mRNA expression was positively correlated with malignant tumor growth factors, including Ki67 $(P<0.001$, Figure 1E) and PCNA $(P<$ 0.001 , Figure 1F), respectively. Furthermore, Gene Set Enrichment Analysis (GSEA) exhibited that CBX3 overexpression was negatively correlated with survival up (Figure 1G) and positively correlated with survival down (Figure 1H) in the TCGA-LIHC dataset.

Kaplan-Meier analysis demonstrated that high expression level of $\mathrm{CBX} 3$ predicted a short overall survival $(P<0.001$, Figure 2A) and a short progression free survival $(P<0.001$, Figure 2B) in HCC patients. When further stratified by TNM stage synchronously, high expression of CBX3 is also a poor prognostic marker for overall survival (Figure 2C) and progression free survival (Figure 2D) for HCC patients, regardless of

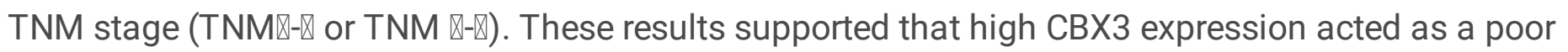
prognostic marker for HCC patients.

\section{Biological functions of CBX3 in HCC cells}

To illustrate the functional role of $\mathrm{CBX} 3$ on the development of HCC cells in vitro. We first conducted qRTPCR and western blot analysis and results showed that the expression level of CBX3 mRNA and protein were really lower in HCC cell lines (Herp-3B and SMMC-7721 cells) than that in normal liver cell lines (L02 and Changliver cells), respectively (Figure 3A). Furthermore, we constructed Herp-3B and SMMC-7721 cells with knockdown the expression of $\mathrm{CBX} 3$ by using RNA interference. The knockdown effect of CBX3 was affirmed by western blotting (Figure 3B). The CCK-8 assayrevealed that the viability of Herp-3B (Figure 3C) and SMMC-7721 (Figure 3D) cells with knockdown of CBX3 was lower than that of control. The wound healing assay showed that $\mathrm{CBX} 3$ knocknown repressed the migration of HCC cells (Figure 3E). Depression of KIF26B expression reduced colony formation and invasiveness capacity of HCC cells by colony formation (Figure 3F) and transwell (Figure 3G) assay.

On the other hand, Herp-3B and SMMC-7721 cells were selected to transfect with CBX3 overexpression plasmids and empty vector, respectively. The efficiency of CBX3 overexpression was confirmed in HCC 
cells by western blotting (Figure 4A). The CKK-8 assay (Figure 4B) showed that increased expression of CBX3 accelerated HCC cell proliferation. Wound healing assay revealed that overexpression of CBX3 facilitated the movement of SMMC-7721 and Herp-3B cells towards the center of the wound (Figure 4C). The colony formation assay showed that the number of colony formation was increased in CBX3 overexpressing group (Figure 4D). The transwell assay exhibited that the invasive ability of SMMC-7721 and Herp-3B cells with overexpressing CBX3 was enhanced compared with that of control (Figure 4E). Collectively, these findings demonstrated that CBX3 was involved in accelerating the biological behaviors of HCC cells.

\section{CBX3 may be involved in the regulation of cell cycle progression of HCC}

To explore the potential mechanism of CBX3's aggressive activity in HCC, Interestingly, KEGG pathway analysis illustrated that the altered majority genes were related to the cell cycle regulation (Figure 5A). GSEA showed that CBX3 expression was positively correlated with the cell cycle progression (Figure 5B). GSVA analysis showed the differential expression genes was related to Myc targets, E2F targets, G2M checkpoint, which were associated with genes in the pathways regulating cell cycle (Figure 5C). Then, we overlapped the alteration genes from KEGG analysis and those from GSVA analysis, E2F, Myc, CDK1 and Cyclin B1 were choosed. To further confirm the major genes correlated with the functional role of CBX3, western blotting was performed to show that the protein expression levels of E2F, Myc, CDK1 and Cyclin B1 were all descended in CBX3 knocking down HCC cells, an opposite effect was found in HCC cells overexpressing CBX3 (Figure 5D). Therefore, CBX3 may participate in HCC development through regulating the cell cycle progression.

\section{CBX3 was a direct target of miR-139 in HCC}

To disclose the upstream mechanism responsible for CBX3 mediated HCC progression. Starbase was used to predict that miR-139 was predicted to be incompletely complementary to CBX3 (Figure 6A). To elucidate whether miR-139 could directly bind to 3'-UTR of CBX3, double luciferase reporter assays were performed to show that luciferase activity in SMMC-7721 and Herp-3B cells carried the wild-type (WT)CBX3 3'-UTR was inhibited by miR-139 overexpression compared with that carried a mutant (Mut) - CBX3 $3^{\prime}$-UTR (Figure 6B). Furthermore, using western blot and qRT-PCR, the expression of CBX3 protein (Figure 6C) and mRNA (Figure 6D) were both markedly decreased in SMMC-7721 and Herp-3B cells when transfected with miR-139 mimics compared with the negative control. Controversially, inhibiting miR-139 expression in these cells increased the expression of CBX3. Overall, the data suggested that CBX3 is a direct target of miR-139 in HCC. Subsequently, analysis of TCGA dataset implied that miR-139 mRNA expression was negatively correlated with CBX3 (Figure 6E). To demonstrate the relationship between miR-139 expression and prognosis in HCC patients, log rank test was utilized to show that miR-139 expression was positively correlated to the favorite overall survival (Figure 6F) and progression free survival (Figure 6G) in HCC patients, regardless of CBX3 expression.

\section{MiR-139 regulated HCC progression through directly targeting CBX3}


That miR-139 could inhibit the growth of HCC cells in cell experiments have been shown in previous studies [11-13]. To assess that miR-139 is a functional direct regulator of CBX3 in HCC cells, miR-139 mimics or miR-NC was transfected with SMMC-7721 and Herp-3B cells, followed by the combined effects of miR-139 and CBX3 overexpression were then investigated, the expression of CBX3 protein (Figure 7A) in the miR-139 mimics group was dramatically decreased relative to that in the miR-NC group, and restored in the combined miR-139 and CBX3 overexpression group. Thereafter, further experiments demonstrated that overexpression of miR-139 alone attenuated the expression levels of cell cycle progression-related proteins (Figure 7G), decreased cell viability and colony-forming ability and prohibited migration and invasion (Figure 7B-F) (all $P<0.05)$ in HCC cells, which were all significantly antagonized by synchronously overexpressing miR-139 and CBX3 (Figure 7A-G). Taken together, our data validated that miR-139 suppressed the progression of HCC partly by regulation of CBX3.

\section{Discussion}

Epigenetic regulation can affect gene transcriptional activity by histone modification without changing DNA sequence [14]. Aberrant epigenetic alteration is a hallmark of cancers[15], and HP1 is an important regulation factor for histone modification involving in DNA damage repairing[16], heterochromatin formation, transcription regulation, and chromatin remodeling. CBX3 encodes a protein called HP1 $\gamma$, which is a member of HP1. CBX3/HP1y was reported to play a tumor-promoting action and may be a therapeutic target in many cancers. Previous studies reported that $\mathrm{CBX} 3$ has a high expression in prostate cancer[17], non-small cell lung cancer (NSCLC)[18], osteosarcoma[19], tongue squamous cell carcinoma (TSCC)[20], colorectal cancer [21]and pancreatic cancer[22]. In addition, MA CHAO et al demonstrated that CBX3 overexpression is a poor predictor for prognosis in osteosarcoma patients [19]. HUAYONG ZHANG et al identified that $\mathrm{CBX} 3 / \mathrm{HP} 1 \mathrm{y}$ upregulation is correlated with a dismal prognosis for tongue squamous cell carcinoma patients. Further cell experiments showed that $\mathrm{CBX} 3$ repression inhibited while CBX3 overexpression promoted the TSCC cell growth and colony formation ability[23]. Recently, Xiaoping Zhong et al reported that $\mathrm{CBX} 3 / \mathrm{HP1}$ y played an important role in $\mathrm{HCC}$ tumorigenerity that might be a poor biomarker for prognosis for HCC patients[24].

In our study, we first acquired the expression levels of CBX3 mRNA in HCC from the public databases. Analysis of the public data showed that CBX3 was significantly upregulated in HCC tissues relative to normal liver tissues. Subsequently, the consistent result was further supported by qRT-PCR and western blot in HCC cell lines, and IHC staining in HCC tissues, respectively. Analysis of TMA investigated that high CBX3 expression was closely correlated to some malignant clinic-pathological characteristics. The log-rank test revealed that high CBX3 expression usually predicted a poor prognosis in HCC patents. In vitro experiments showed that knocking down $\mathrm{CBX} 3$ could result in repressing the growth, migration and invasive ability of SMMC-7721 and Herp-3B HCC cells, however, overexpressing CBX3 promoted the progression of $\mathrm{HCC}$ cells. Our results investigated that $\mathrm{CBX} 3$ was involved in the progression and may play as a dismal prognostic factor in HCC. 
Disruptions in cell cycle regulation play important roles in HCC progression [25, 26]. Previous research revealed that $\mathrm{CBX} 3$ was involved in the progression of multiple cancers through regulating cell cycle regulation $[20,27]$. It was reported that knockdown of $C B X 3$ expression prohibited cells proliferation via the increase in G0/G1 phases arrest and apoptosis [19]. Zhang HuaYong et al illustrated that CBX3 accelerated TSCC cell growth through G1/S phase modulation by suppressing expression of p21[23]. Liu Ming et al demonstrated that HP1y promoted the proliferation of colorectal cancer cells in vitro and in vivo by targeting p21 directly[21]. To explore the mechanism of how CBX3 regulated HCC progression. Analysis of KEGG pathway and GSEA revealed that CBX3 expression alteration is significantly correlated with cell cycle progression. GSVA showed that $\mathrm{CBX} 3$ was positively associated with MYC targets, E2F targets and G2/M checkpoint.Western blotting proved that HP1 protein was positively correlated to the key transcriptional regulators of cell cycle regulation, such as E2F, Myc, CDK1 and Cyclin B1. Our data showed that $\mathrm{CBX} 3$ was involved in HCC development by regulating cell cycle progression.

In recent years, miRNAs have been considered to play as tumor-promoting action or tumor-suppressing action in various tumors $[28,29]$. CBX3 has been reported to be regulated by miR-30a in colorectal cancer. Otherwise, HP1Y functions as an oncogene which directly repressed miR-451a expression in prostate cancer progression [17]. However, upregulation of HP1y may be regulated by kinds of miRNAs in HCC. According to the bioinformatics analysis, miR-139 was predicted to be the target regulator of CBX3 in HCC. Then, the target interplay between miR-139 and CBX3 was verified by dual-luciferase reporter assay. The results of qRT-PCR and Western blot showed that miR-139 expression is negatively correlated with CBX3 expression. Confirmatory experiments showed that repressed expression of CBX3 could reverse the proliferation and invasion promoting by miR-139 downregulation. The data revealed that miR-139 functioned as a tumor suppressor involving in HCC progression, which is consistent with the results of previous reports $[12,30,31]$. WONG CARMEN CHAK-LUI et al demonstrated that miR-139 is downregulated in HCC and played as a tumor suppressing role in inhibiting [12]. Moreover, Zan Ying et al reported that miR-139 overexpression led to decreased c-myc in HCC[32]. Our results also showed that miR-139 expression is negatively related with the expression of E2F, Myc, CDK1 and Cyclin B1 detected by western blot. And rescue experiments exhibited that miR-139 inhibited HCC progession through direct prohibition of CBX3 expression.

Collectively, the present study demonstrated that CBX3 served as a tumor-promoting molecular role in HCC development and a poor prognostic biomarker for HCC patients. We found that miR-139 may play an anticancer role by inhibiting the expression of CBX3 in HCC. Our results identified that the miR-139/CBX3 axis may involve in the HCC development through regulating cell cycle progression and may be a promising target in the treatment of HCC.

\section{Declarations}

Ethics approval and consent to participate: 
This study obtained the approval of the Ethics Committee of Luoyang Central Hospital Affiliated to Zhengzhou University. The research has been carried out in accordance with the World Medical Association Declaration of Helsinki. All of the patients were consent to participate in this study.

Consent for publication:

Yes.

Availability of data and materials:

The datas used to support the findings of this study and related materials are available from the corresponding author upon request.

Competing interests:

The authors declare that they have no competing interests.

Fundings:

This work was supported by the Henan Medical Science and Technology Public Relations Project (Grant No. 2018020914) and the Foundation of Luoyang Central Hospital (Grant No. LHGJ20191222).

Authors' contributions:

Guotao Li conceived the experiments; Xiaoyan Yang gave an experimental guidance in the lab; Zhongming Zha andYumeng Zhu performed the experiments; Guoqiang Zhang designed the experiments, Pan Zhang analyzed the data and wrote the paper.

Acknowledgements:

None.

\section{References}

1. Poon D, Anderson BO, Chen LT, Tanaka K, Lau WY, Van Cutsem E, Singh H, Chow WC, Ooi LL, Chow P et al: Management of hepatocellular carcinoma in Asia: consensus statement from the Asian Oncology Summit 2009. Lancet Oncol 2009, 10(11):1111-1118.

2. Ferlay J, Colombet M, Soerjomataram I, Mathers C, Parkin DM: Estimating the global cancer incidence and mortality in 2018: GLOBOCAN sources and methods. 2019, 144(8):1941-1953.

3. Dhanasekaran R, Nault JC, Roberts LR, Zucman-Rossi J: Genomic Medicine and Implications for Hepatocellular Carcinoma Prevention and Therapy. Gastroenterology 2019, 156(2):492-509. 
4. EASL Clinical Practice Guidelines: Management of hepatocellular carcinoma. J Hepatol 2018 , 69(1):182-236.

5. Smallwood A, Hon GC, Jin F, Henry RE, Espinosa JM, Ren B: CBX3 regulates efficient RNA processing genome-wide. Genome Res 2012, 22(8):1426-1436.

6. Akaike Y, Kuwano Y, Nishida K, Kurokawa K, Kajita K, Kano S, Masuda K, Rokutan K: Homeodomaininteracting protein kinase 2 regulates DNA damage response through interacting with heterochromatin protein 1Y. Oncogene 2014, 34(26):3463-3473.

7. Rupaimoole R, Slack FJ: MicroRNA therapeutics: towards a new era for the management of cancer and other diseases. Nat Rev Drug Discov 2017, 16(3):203-222.

8. Hayes J, Peruzzi PP, Lawler S: MicroRNAs in cancer: biomarkers, functions and therapy. Trends Mol Med 2014, 20(8):460-469.

9. Zhu W, Li H, Yu Y, Chen J, Chen X, Ren F, Ren Z, Cui G: Enolase-1 serves as a biomarker of diagnosis and prognosis in hepatocellular carcinoma patients. Cancer Manag Res 2018, 10:5735-5745.

10. Bao J, Yu Y, Chen J, He Y, Chen X, Ren Z, Xue C, Liu L, Hu Q, Li J et al: MiR-126 negatively regulates PLK-4 to impact the development of hepatocellular carcinoma via ATR/CHEK1 pathway. Cell Death Dis 2018, 9(10):1045.

11. Yang $L$, Yin D, Wang Y, Cao L: Inhibition of the growth of hepatocellular carcinoma cells through fibroblast growth factor 18 suppressed by miR-139. Oncol Rep 2017, 38(4):2565-2571.

12. Wong CC, Wong CM, Tung EK, Au SL, Lee JM, Poon RT, Man K, Ng IO: The microRNA miR-139 suppresses metastasis and progression of hepatocellular carcinoma by down-regulating Rho-kinase 2 . Gastroenterology 2011, 140(1):322-331.

13. Gu W, Li X, Wang J: miR-139 regulates the proliferation and invasion of hepatocellular carcinoma through the WNT/TCF-4 pathway. Oncol Rep 2014, 31(1):397-404.

14. Sridharan R, Gonzales-Cope M, Chronis C, Bonora G, McKee R, Huang C, Patel S, Lopez D, Mishra N, Pellegrini $\mathrm{M}$ et al: Proteomic and genomic approaches reveal critical functions of H3K9 methylation and heterochromatin protein-1 gamma in reprogramming to pluripotency. Nat Cell Biol 2013, 15(7):872-882.

15. Hanahan D, Weinberg RA: Hallmarks of cancer: the next generation. Cel/ 2011, 144(5):646-674.

16. Dialynas GK, Vitalini MW, Wallrath LL: Linking Heterochromatin Protein 1 (HP1) to cancer progression. Mutat Res 2008, 647(1-2):13-20.

17. Chang C, Liu J, He W, Qu M, Huang X, Deng Y, Shen L, Zhao X, Guo H, Jiang J et al: A regulatory circuit HP1gamma/miR-451a/c-Myc promotes prostate cancer progression. Oncogene 2018, 37(4):415-426. 
18. Chang SC, Lai YC, Chen YC, Wang NK, Wang WS, Lai JI: CBX3/heterochromatin protein 1 gamma is significantly upregulated in patients with non-small cell lung cancer. Asia Pac J Clin Oncol 2018, 14(5):e283-e288.

19. Ma C, Nie XG, Wang YL, Liu XH, Liang X, Zhou QL, Wu DP: CBX3 predicts an unfavorable prognosis and promotes tumorigenesis in osteosarcoma. Mol Med Rep 2019, 19(5):4205-4212.

20. Zhang H, Chen W, Fu X, Su X, Yang A: CBX3 promotes tumor proliferation by regulating G1/S phase via p21 downregulation and associates with poor prognosis in tongue squamous cell carcinoma. Gene 2018, 654:49-56.

21. Liu M, Huang F, Zhang D, Ju J, Wu XB, Wang Y, Wang Y, Wu Y, Nie M, Li Z et al: Heterochromatin protein HP1gamma promotes colorectal cancer progression and is regulated by miR-30a. Cancer Res 2015, 75(21):4593-4604.

22. Chen LY, Cheng CS, Qu C, Wang P, Chen H, Meng ZQ, Chen Z: CBX3 promotes proliferation and regulates glycolysis via suppressing FBP1 in pancreatic cancer. Biochem Biophys Res Commun 2018, 500(3):691-697.

23. Zhang H, Fu X, Su X, Yang A: CBX3/HP1gamma is upregulated in tongue squamous cell carcinoma and is associated with an unfavorable prognosis. Exp Ther Med 2018, 15(5):4271-4276.

24. Zhong X, Kan A, Zhang W, Zhou J, Zhang H, Chen J, Tang S: CBX3/HP1gamma promotes tumor proliferation and predicts poor survival in hepatocellular carcinoma. Aging (Albany NY) 2019, 11(15):5483-5497.

25. Klungboonkrong V, Das D, McLennan G: Molecular Mechanisms and Targets of Therapy for Hepatocellular Carcinoma. J Vasc Interv Radiol 2017, 28(7):949-955.

26. Shen S, Dean DC, Yu Z, Duan Z: Role of cyclin-dependent kinases (CDKs) in hepatocellular carcinoma: Therapeutic potential of targeting the CDK signaling pathway. Hepatol Res 2019.

27. Chen LY, Cheng CS, Qu C, Wang P, Chen H, Meng ZQ, Chen Z: Overexpression of CBX3 in Pancreatic Adenocarcinoma Promotes Cell Cycle Transition-Associated Tumor Progression. Int J Mol Sci 2018, 19(6).

28. Rossi JJ: New hope for a microRNA therapy for liver cancer. Cell 2009, 137(6):990-992.

29. Ling H, Fabbri M, Calin GA: MicroRNAs and other non-coding RNAs as targets for anticancer drug development. Nat Rev Drug Discov 2013, 12(11):847-865.

30. Chen JA, Yu Y, Xue C, Chen XL, Cui GY, Li J, Li KF, Ren ZG, Sun RR: Low microRNA-139 expression associates with poor prognosis in patients with tumors: A meta-analysis. Hepatobiliary Pancreat Dis Int 2019, 18(4):321-331. 
31. Li P, Xiao Z, Luo J, Zhang Y, Lin L: MiR-139-5p, miR-940 and miR-193a-5p inhibit the growth of hepatocellular carcinoma by targeting SPOCK1. J Cell Mol Med 2019, 23(4):2475-2488.

32. Zan Y, Wang B, Liang L, Deng Y, Tian T, Dai Z, Dong L: MicroRNA-139 inhibits hepatocellular carcinoma cell growth through down-regulating karyopherin alpha 2. Journal of Experimental \& Clinical Cancer Research 2019, 38(1).

Figures 

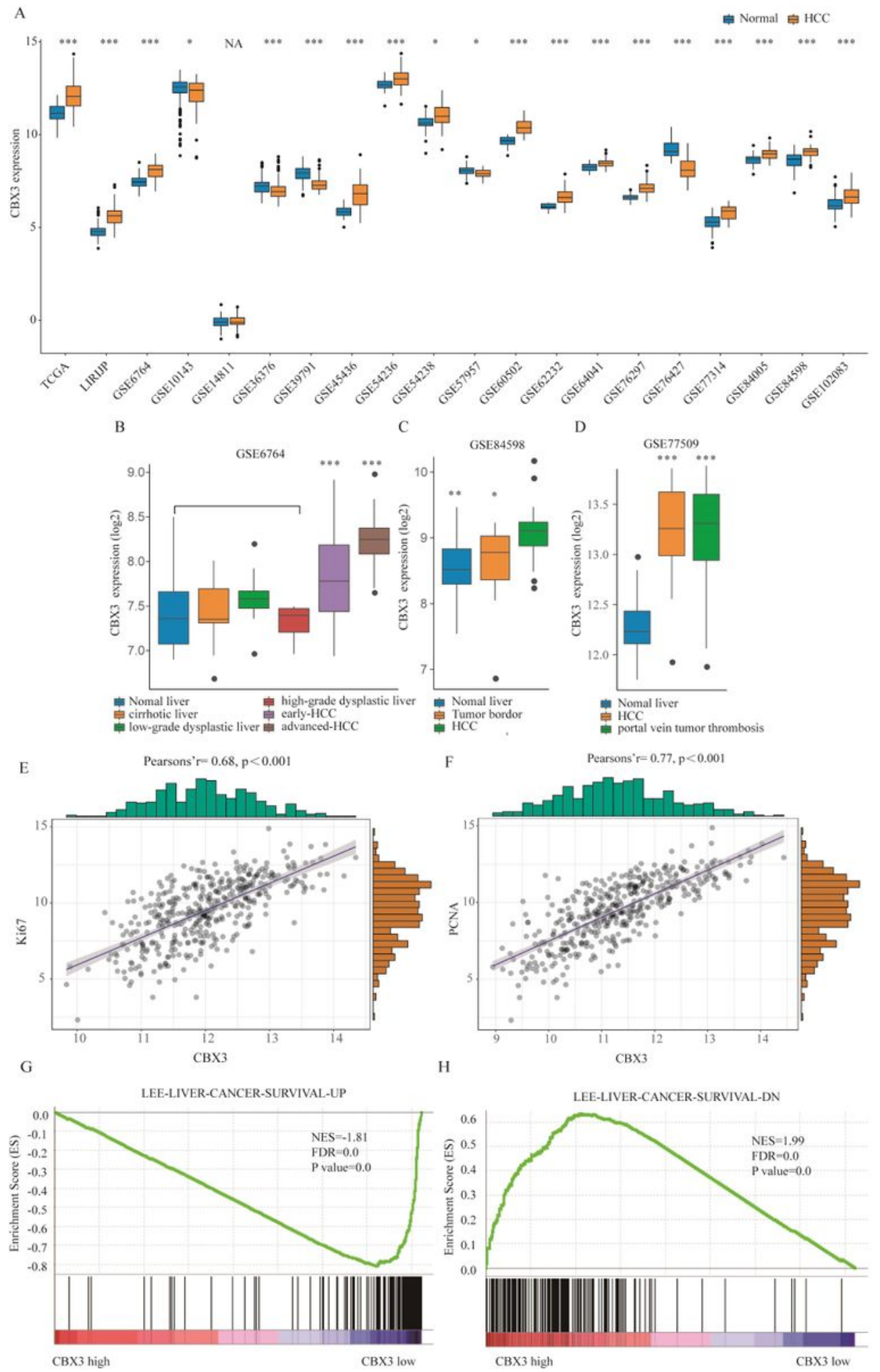

\section{Figure 1}

CBX3 mRNA was upregulated in HCC tissues and was positively correlated to malignant pathological features of HCC (A) Data of TCGA, LIRIJP and GEO database showed that the expression levels of CBX3 mRNA were tendency really high in HCC tissues. $(B, C) C B X 3$ mRNA expression was overexpressed in HCC than that in non-tumor liver tissues. (D) Elevated CBX3 expression in HCC and portal vein tumor thrombosis.(E,F) Spearman's correlation showed that CBX3 mRNA expression was positively correlation 
with Ki67 (E) and PCNA (F) in HCC patients, respectively. (G, H) GSEA analysis showed that CBX3 mRNA expression was positively correlated with liver cancer survival down $(G)$ and negatively associated with liver cancer survival up $(\mathrm{H})$.

A

B
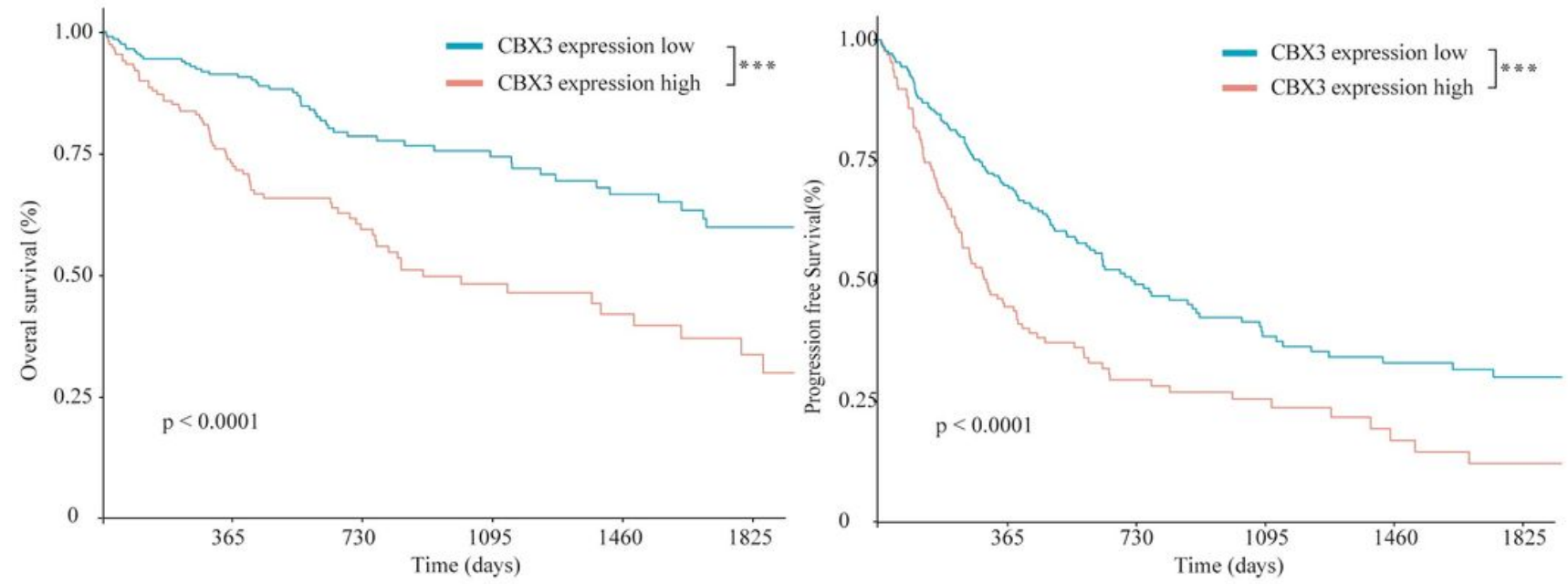

C

D
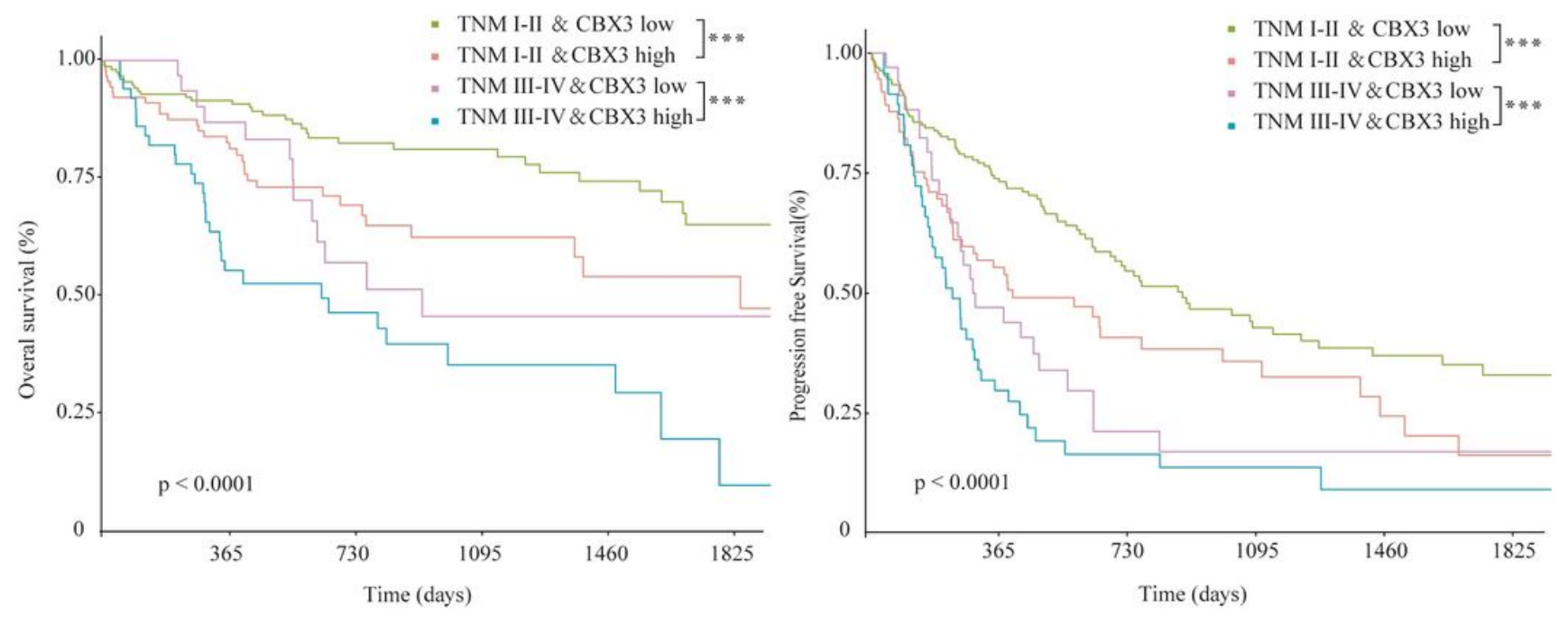

\section{Figure 2}

High CBX3 expression was a poor prognostic biomarker for HCC patients (A, B) Kaplan-Meier analysis exhibited that overexpression of $C B X 3$ was correlated to poor overall survival $(O S, A)$ and progression free survival (PFS, B) for HCC patients from analysis of the TCGA LIHC database. (C, D) Regardless of the

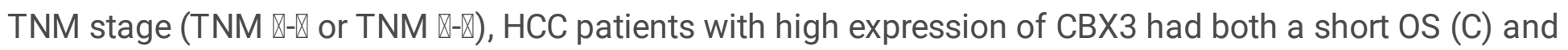
a short PFS (D). 

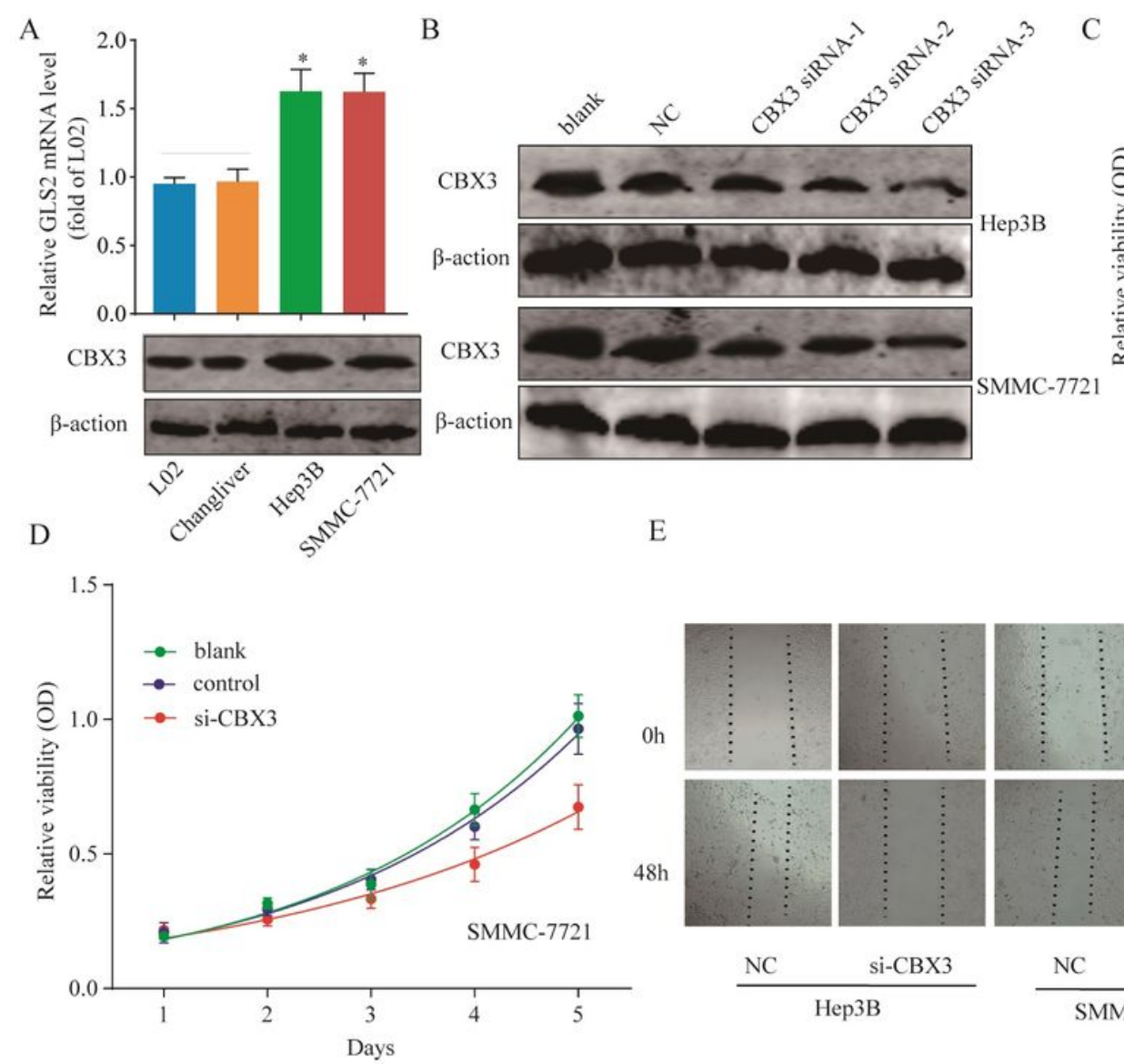

F

$$
=\mathrm{NC}
$$$$
=\mathrm{si}-\mathrm{CBX} 3
$$

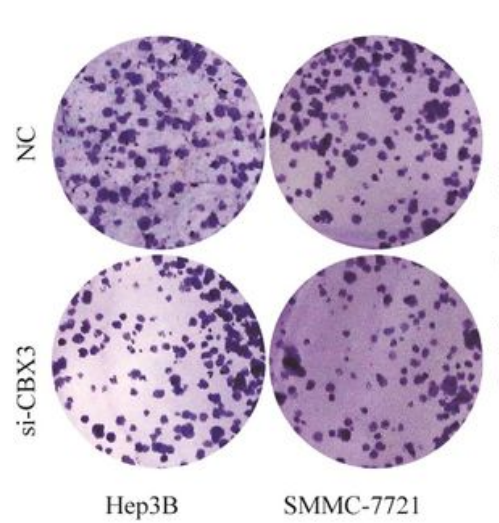

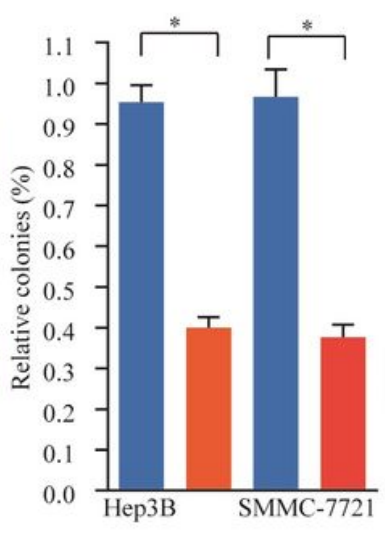

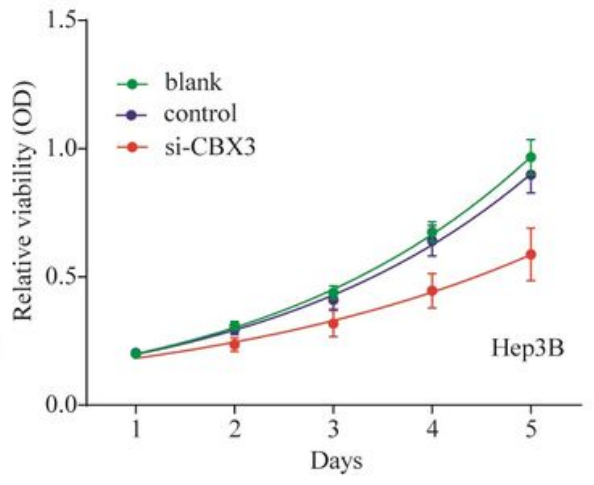

- NC

- $\mathrm{si-CBX3}$

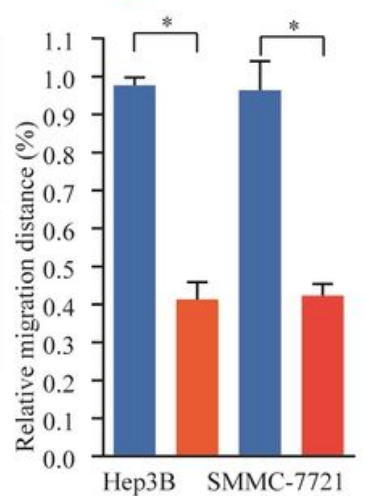

G

$=\mathrm{NC}$

$=\mathrm{si}-\mathrm{CBX} 3$

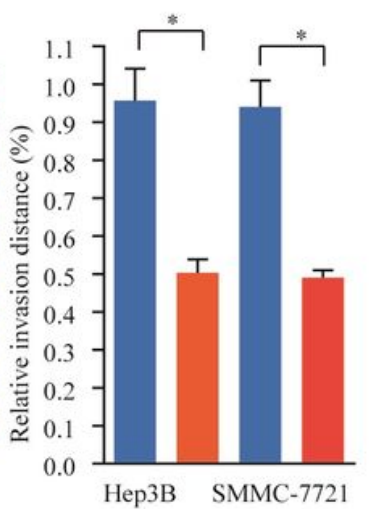

\section{Figure 3}

Inhibition of CBX3 suppressed the biological behaviors of HCC cells (A) CBX3 was overexpressed in HCC cell lines compared with that in the normal liver cell lines, which examined by qRT-PCR and Western blot.

(B) The knockdown efficiency of CBX3 siRNAs in Hep3B and SMMC-7721 cells was measured by Western blot. (C, D) MTT assay showed that the viability of Hep3B (C) and SMMC-7721 cells (D) was decreased by inhibition of CBX3. (E) Wound healing assay indicated that CBX3 knocking down inhibited Hep3B and SMMC-7721 cell migration. (F) Colony numbers of CBX3 knocking down HCC cells were significantly 
reduced compared to respective NC. (G) Transwell assay showed that the invasion capability of HCC cells was attenuated by CBX3 knockdown.
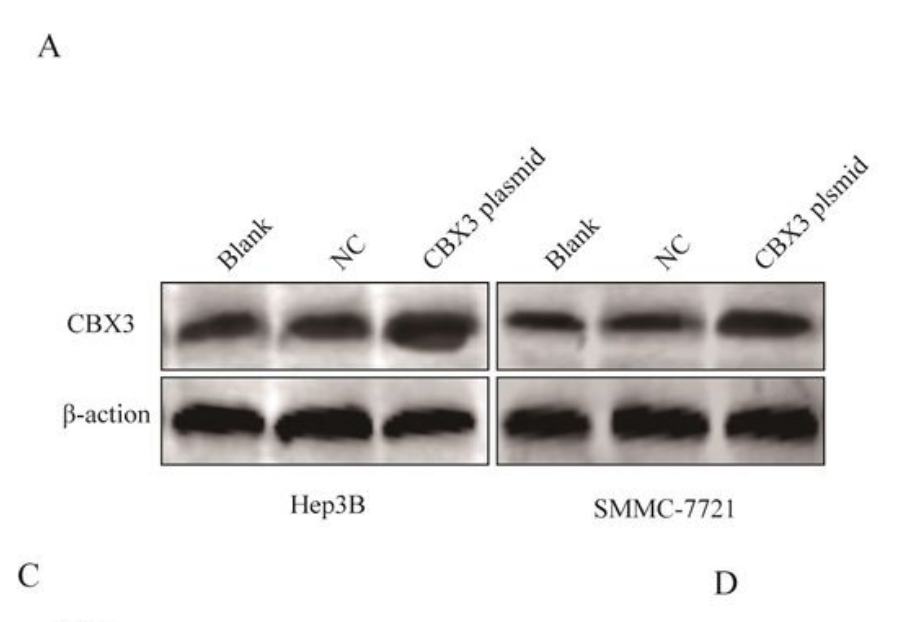

D
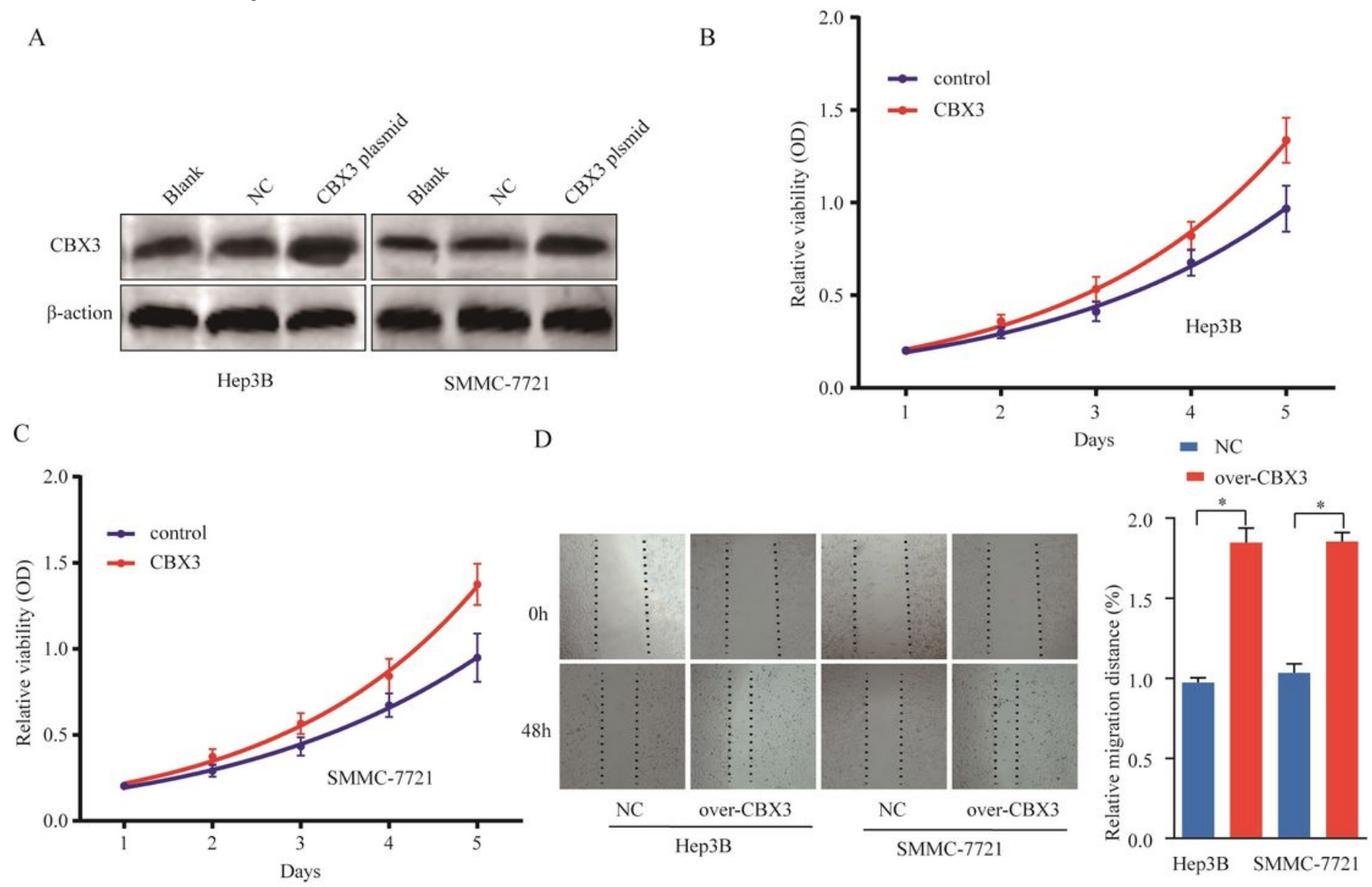

E

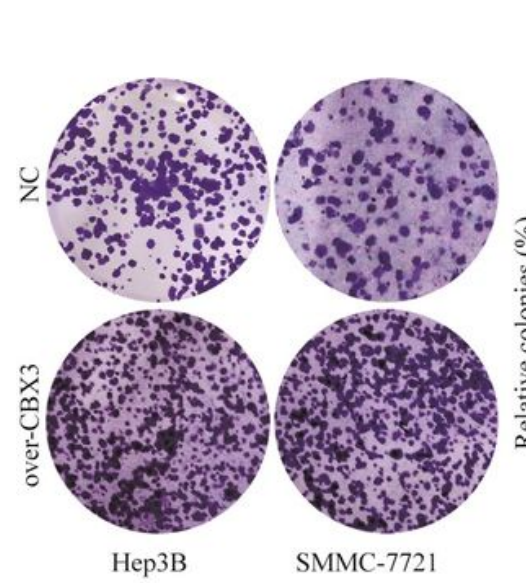

$$
\text { - NC }
$$$$
\text { - over-CBX3 }
$$

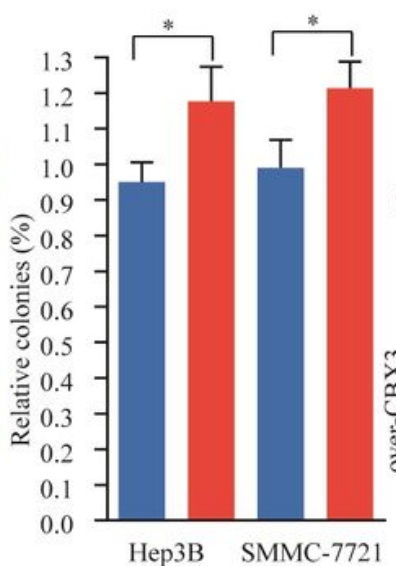

$\mathrm{F}$

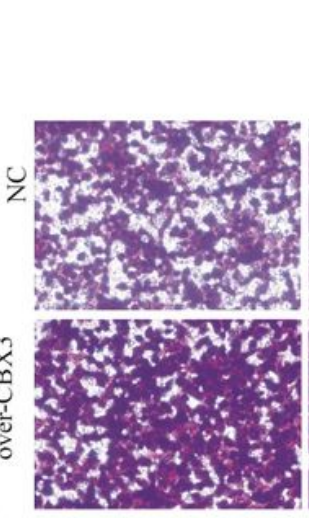

Hep3B

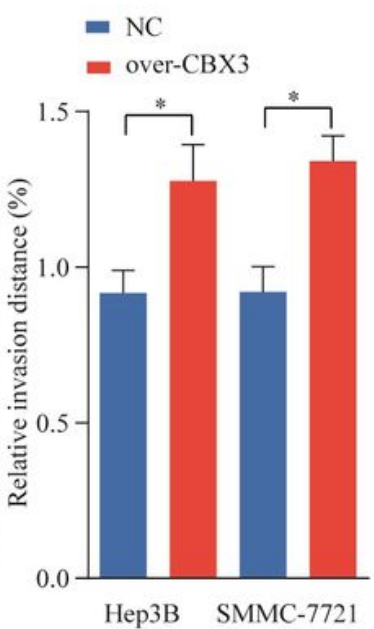

\section{Figure 4}

Overexpression of CBX3 played a tumor-promoting role in HCC progression (A) Hep3B and SMMC-7721 cells transfected with pcDNA3.1-CBX3 or pcDNA3.1-vector, assessed for CBX3 expression by western blot. $(B, C)$ CCK-8 assays were carried out to examine the effects of $C B X 3$ overexpression on the proliferative ability of Hep3B (B) and SMMC-7721 (C) cells. (D) Wound-healing assays were performed to determine 
the effects of CBX3 overexpression on the migration of HCC cells. (E) Colony formation assays in HCC cells after the overexpression of $\mathrm{CBX} 3$. (F) The invasion assay was used to examine the effects of $\mathrm{CBX} 3$ overexpression on HCC cells invasion. ${ }^{*} \mathrm{P}<0.05$.

A

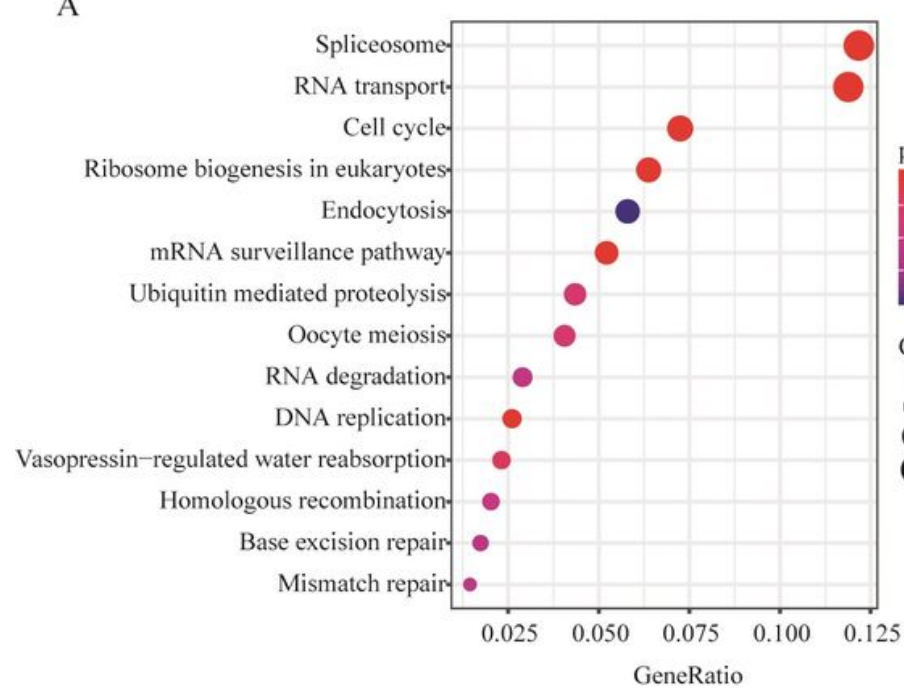

$\mathrm{C}$

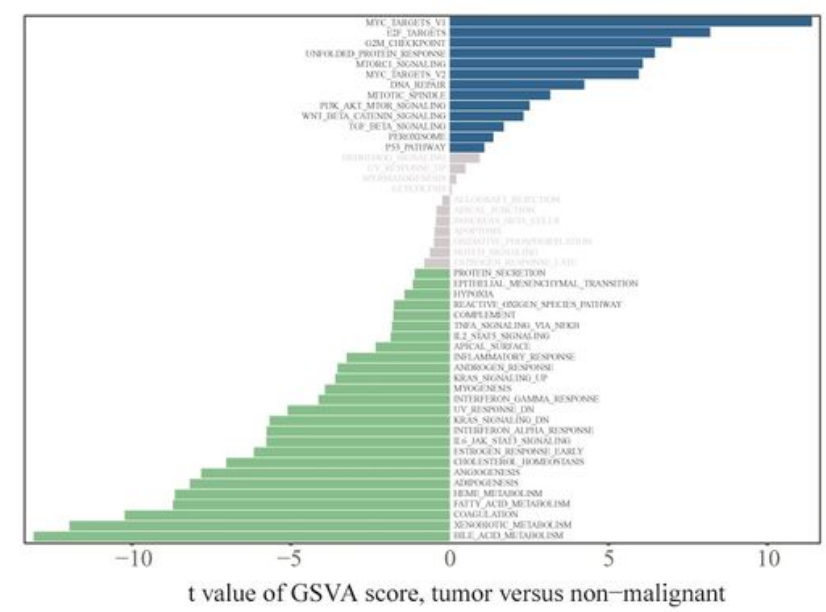

B

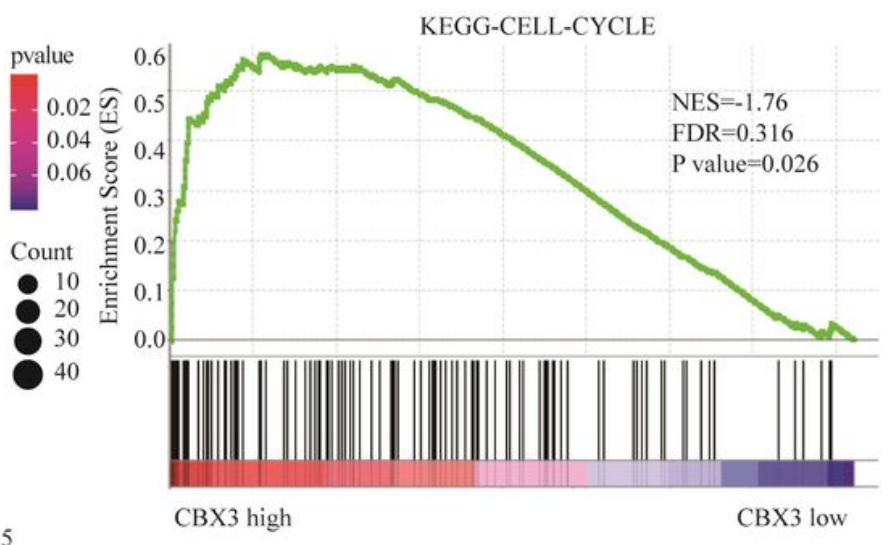

$\mathrm{D}$

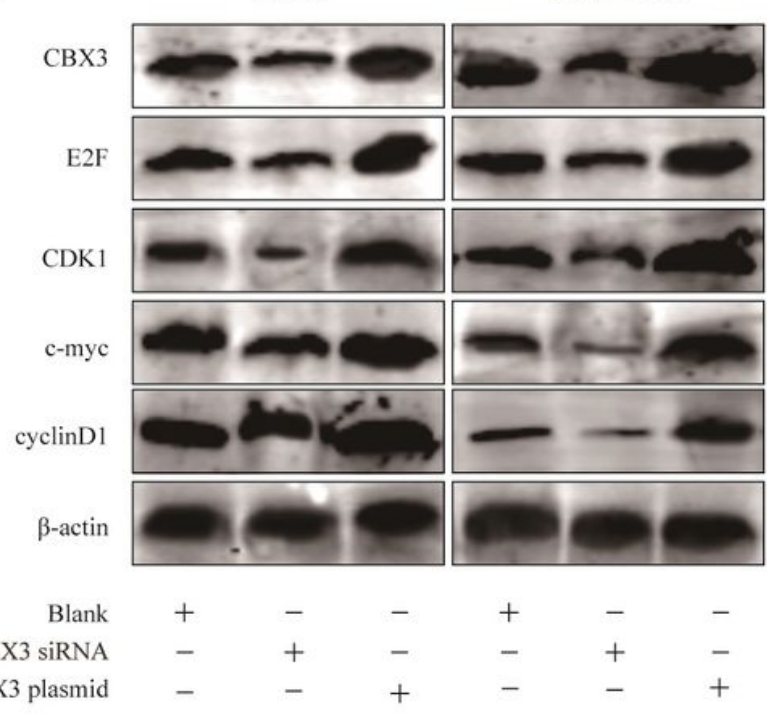

\section{Figure 5}

CBX3 regulates HCC progression through cell cycle regulation (A, B, C) Crossed analysis of KEGG (A), GSEA (B) and GSVA (C) showed that CBX3 expression was correlated to cell cycle regulation. (D) Western blot analysis was used to detect the level of E2F, C-Myc, CDK1 and Cyclin B1 in indicated cells, $\beta$-actin as a loading control. 
A

$5 . A$ A

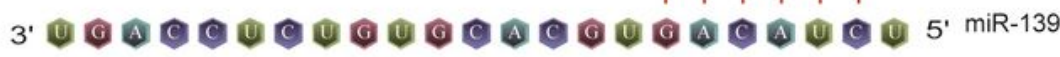

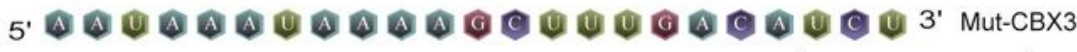
C

CBX3

$\beta$-action

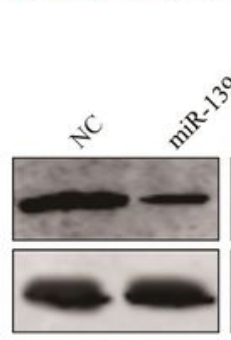

Нер3В

- Blank

D

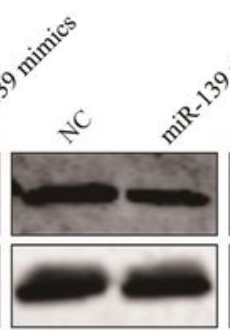

SMMC-7721

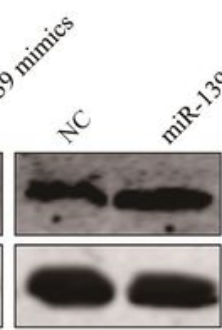

Нер3В

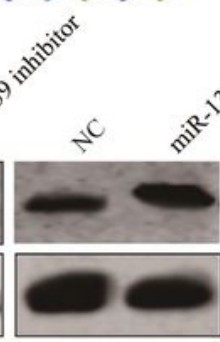

SMMC-7721
E

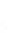

B

$$
\begin{array}{ll}
=\mathrm{Wt}+\mathrm{NC} & =\mathrm{Mut}+\mathrm{NC} \\
=\mathrm{Wt}+\mathrm{miR}-139 & =\text { Mut+miR-139 }
\end{array}
$$

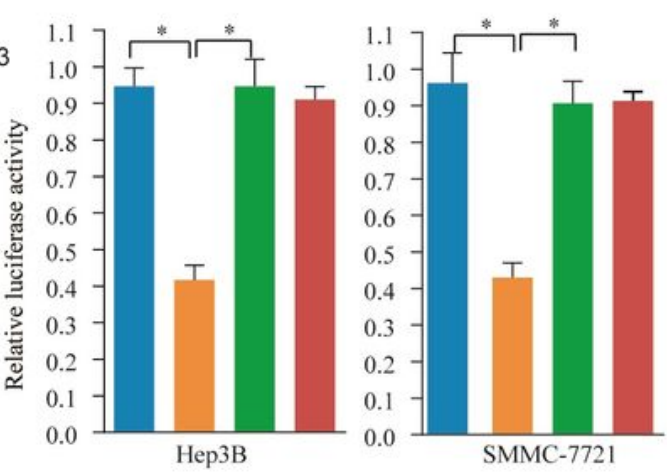

Pearsons' $r=-0.50, p<0.001$
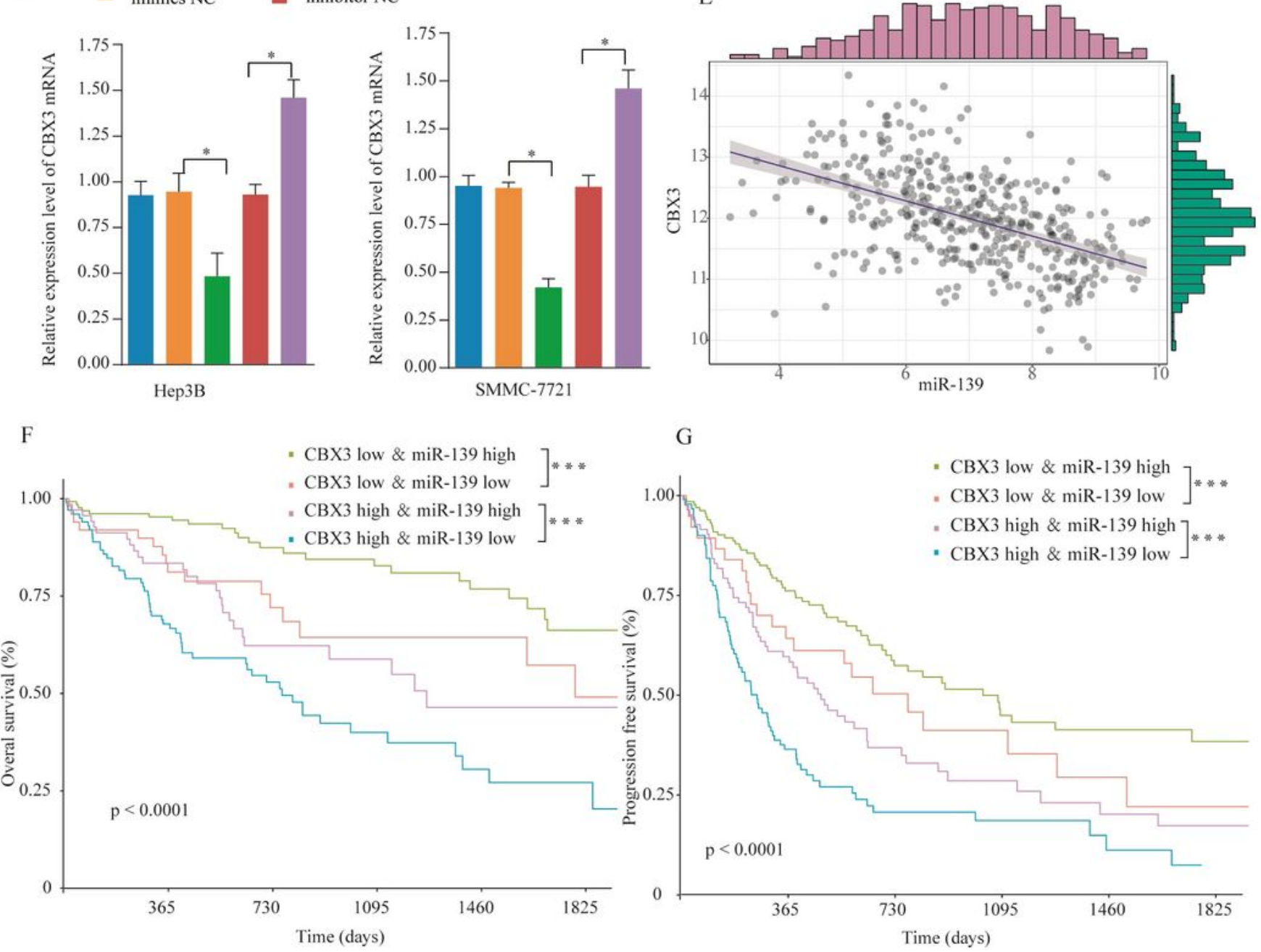

G

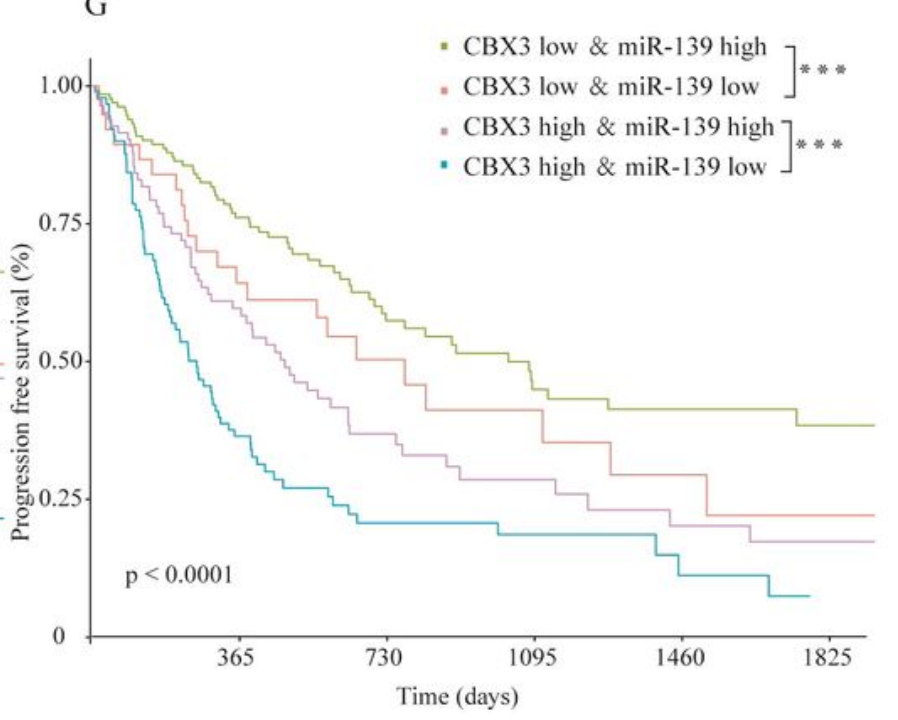

Figure 6

miR-139 directly targeted CBX3 in HCC (A) miR-139 was predicted to be directly binding to 3'UTR of CBX3 and the corresponding mutant sites of CBX3. (B) The luciferase activity of wild-type (Wt) CBX3-3'UTR and mutant (Mut) CBX3-3'UTR was assessed in Hep3B and SMMC-7721 cells. (C, D) After transfecting Hep3B and SMMC-7721 cells with miR-139 mimics or miR-139 inhibitor, Western blotting (C) and RT-qPCR (D) analysis were used to examine the protein and mRNA levels of CBX3. (E) Spearman's analysis revealed 
that miR-139 expression was inversely associated with CBX3 mRNA expression from TCGA database. (F, G) Stratified by both TNM stage and miR-139 expression levels, Kaplan-Meier analysis showed that miR139 expression was positively correlated with the overall survival (OS, F) and progression free survival (PFS, G) of HCC patients. ${ }^{*} P<0.05,{ }^{*} P<<0.01, * \star * P<0.001$.
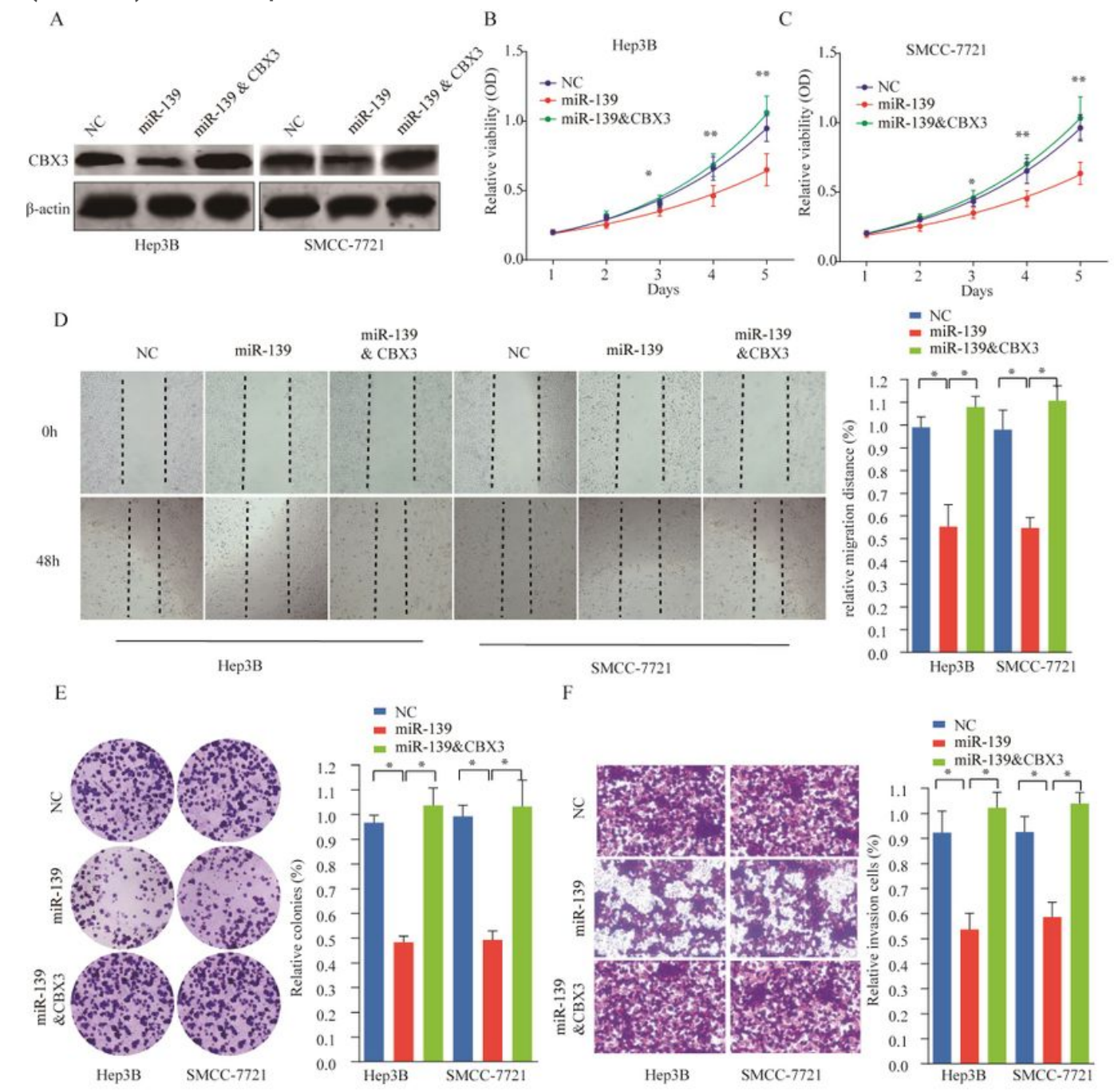

F
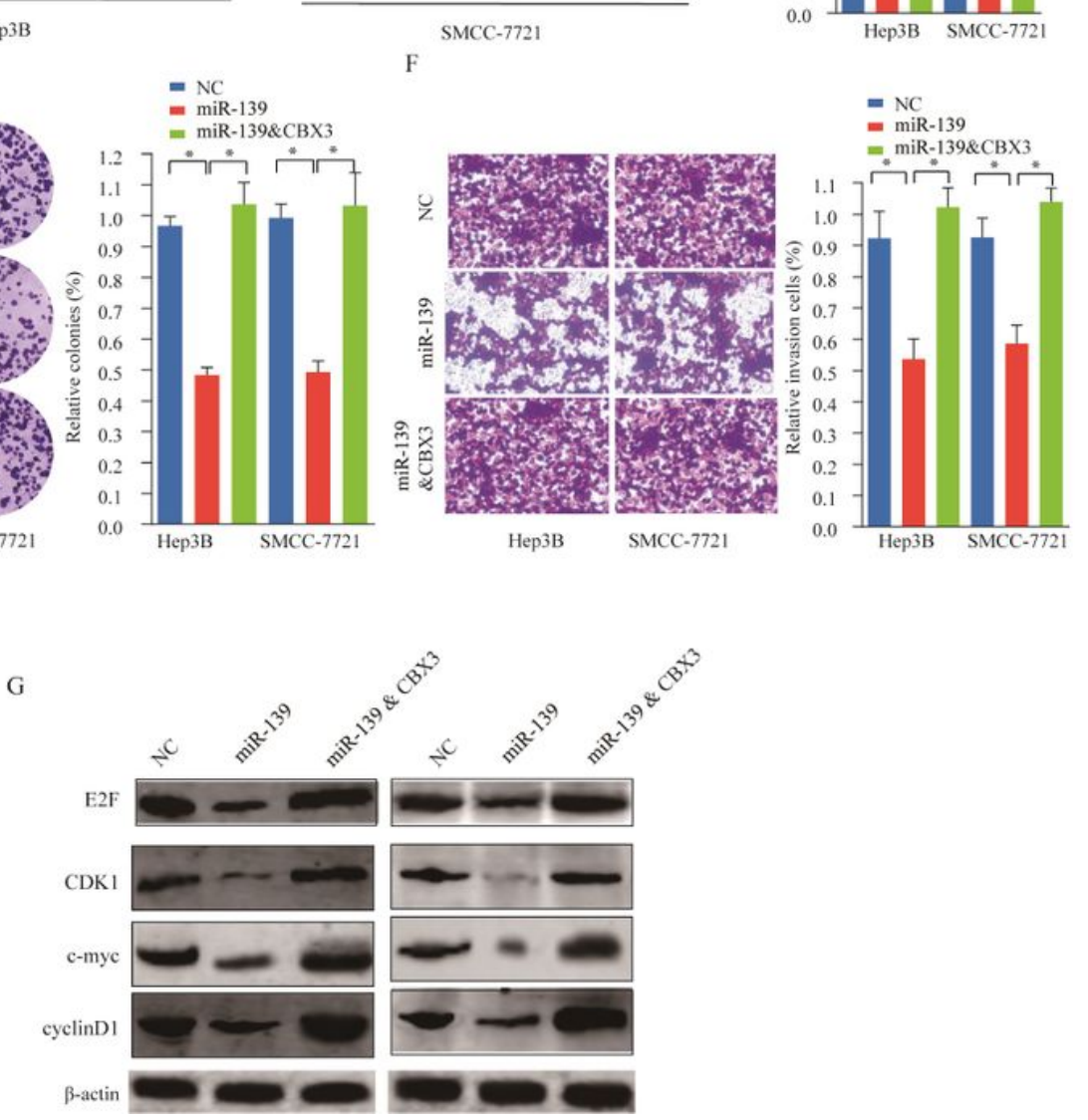

Figure 7

Restoration of CBX3 ameliorates the inhibitory effect of miR-139 in HCC cell lines (A) Hep3B and SMMC7721 cells were transfected with NC, miR-139 mimics or miR-139 mimics $\mathbb{C} B \mathrm{BX} 3$, shown by western blot analysis. (B, C) Cell viability in Hep3B (B) and SMMC-7721 (C) cells examined by CCK-8. (D) Wound- 
healing assays were performed to determine the migration of the introduced Hep3B and SMMC-7721 cells. (E) Colony formation assays. (F) Invading capacities of cells were assayed as described above. (G) Western blot showing the expression of E2F, c-Myc, CDK1 and Cyclin B1 in indicated Hep3B and SMMC7721 cells. ${ }^{\star} \mathrm{P}<0.05,{ }^{\star *} \mathrm{P}<0.01$.

\section{Supplementary Files}

This is a list of supplementary files associated with this preprint. Click to download.

- originaldatas.rar 